\title{
Partially Balanced Cross-Over Designs for Consumer Trials
}

\author{
Poonam Singh $^{1}$, Mithilesh Kumar Jha ${ }^{2}$, Garima Priyadarshini ${ }^{1 *}$ \\ ${ }^{1}$ Department of Statistics, University of Delhi, Delhi-110007, India. \\ ${ }^{2}$ Department of Statistics, PGDAV College (University of Delhi), \\ Nehru Nagar, Delhi-110065. \\ *Corresponding Author: priyadarshinigarima@gmail.com
}

\begin{abstract}
Received: $22^{\text {nd }}$ February 2016 / Revised: $3^{\text {rd }}$ August $2016 /$ Accepted: $14^{\text {th }}$ August 2016 OIAppStat-SL2016
\end{abstract}

\begin{abstract}
In cross-over designs, sequences of treatments are applied to the units, spread over a specified period of time. The model for the estimation of such designs includes a carryover term, which makes them competent for use in consumer research trials. Construction of useful cross-over designs in the context of consumer research trials forms the body of this work and some new classes of partially balanced cross-over designs with two associate classes [PBCOD(2)] are obtained, along with their efficiencies. Designs constructed are uniform over periods for a large number of units and they estimate treatment contrasts with two sets of precision.
\end{abstract}

Keywords: Cross-over design; partially balanced cross-over design; partially balanced design; consumer trials.

\section{Introduction}

Consumer research is a field where a number of individuals are subjected to a sequence of treatments over a period of time with an aim to evaluate some product. The procedure of conduct of such trials makes them vulnerable to residual or carryover effects of the preceding treatment(s), rendering the usual designs of parallel trials incompetent for use in them. Cross-over designs, being tailored for the presence of such carry-over effects, are competent for use in consumer research trials. The situation, where the main purpose of the researcher is to balance the 
carry-over effects in consumer trials when each consumer only receives $k$ out of possible $v$ products, is considered here.

Williams' Latin square designs are used widely in consumer research to plan complete block experiments. Williams (1949) gives a general way of constructing designs that are balanced for first-order carry-over effects and also gives an analysis of variance to estimate these effects. These designs consist of either one or two Latin squares depending on whether the number of treatments being used is even or odd respectively. MacFie, et al. (1989) have pointed out the usefulness of these techniques when carrying out hall tests. Schlich (1993) used data from a sensory experiment on restructured steaks and found significant carry-over effects when Williams' analysis of variance is applied.

Patterson (1952) has shown that both complete and incomplete block designs that use all possible orders of presentation will be completely balanced for all carry-over effects up to $(k-1)$ th order. A requirement to achieve this balance is that all $v(v-1)$ combinations of products occur equally often in any given pair of adjacent or nonadjacent positions in the order of presentation. This simple approach has the obvious disadvantage that large numbers of experimental units are required. However, provided that the experiments may be conducted quickly and cheaply, as can be the case in consumer trials where data are routinely collected from hundreds of people, all possible combinations of designs may be of some considerable value.

Williams (1949) demonstrated that a complete set of MOLS (mutually orthogonal Latin squares) also gives a design that is balanced for all orders of carry-over effects, provided that the first column of each square is identical. Then the $n(n-1)$ orders of presentation are found from the rows of each square, where $n$ is the order of Latin squares.

Sometimes it will not be possible to obtain a multiple of $v(v-1)$ consumers. In these cases it may be preferable to sacrifice the balancing of higher order carry-over effects in order to achieve a design with fewer subjects. There exists a class of designs, which may be easily derived from the Williams designs, balanced for firstorder carry-over effects.

There is a very simple approach due to Patterson (1951) that combines the use of Williams designs balanced for first-order carry-over and standard balanced incomplete block designs (BIBD). For any BIBD with block size $k$, replace each block by a full Williams design of order $k$ constructed with the same treatment symbols as the block of the BIBD. This ensures that all ordered pairs of two 
treatments from the block of BIBD will occur in the rows of the Latin square used to replace it. Since all BIBDs have equal incidences of treatment pairs within blocks, the compound design described must, therefore, be balanced for first-order carry-over effects.

Balanced cross-over designs, uniform over periods that can be used in consumer trials have been discussed extensively in literature. Wakeling and MacFie (1995) gave incomplete designs balanced for first and higher order carry-over effects, Wakeling et. al (2001) presented cyclic designs and Perinel and Pages (2004) gave optimal nested designs, for consumer research trials. Ball (1997) advocated the use of nearly balanced cross-over designs for practical purposes of sensory evaluation, in cases where a balanced design is not possible due to some constraint. Use of such designs in consumer research trials was further taken up by Kunert and Sailor (2006).

Consumer trials are conducted over a large number of units and the treatments, which consist of a series of questions or tasting samples etc., may be such that difference between some of them needs to be obtained with higher precision as opposed to others. Situations like these require designs that span over a large number of units and estimate treatment contrasts with more than one set of precision. As the designs suitable for consumer trials are cross-over designs, a way out in such instances is the use of cross-over designs that spread over a reasonably large number of units and are partially balanced with two associate classes.

Construction of partially balanced cross-over designs with two associate classes $(\operatorname{PBCOD}(2))$, in the context of consumer research trials, forms the body of this work and some new classes of connected $\operatorname{PBCOD}(2)$ with large number of units are obtained, using two associate class association schemes, partially balanced incomplete block designs with two associate classes (PBIBD (2)), orthogonal arrays (OA) and mutually orthogonal Latin squares (MOLS). The variances of the estimated direct and residual effects and their efficiencies are also computed.

The definition, model and efficiency factors for $\operatorname{PBCOD}(2)$ are given in Section 2. The methods of constructing some new classes of $\operatorname{PBCOD}(2)$ are given and illustrated in Section 3, along with the computed variances and efficiencies. Conclusions are presented in Section 4. 


\section{Definition, Model, Variance and Efficiency}

\section{Definition}

A PBCOD, based on an $m$-class association scheme, with parameters $v, k, t, b, \mu_{i}, v_{i}$, $\lambda_{i}(i=1,2, \ldots, m)$, as defined by Blaisdell and Raghavarao (1980), is a $k \times b$ array that possesses the following properties:

1. Every treatment occurs at most once in a column.

2. Every treatment occurs $t$ times in each row.

3. Every pair of treatments $(\theta, \phi)$ occurs together in $\mu_{i}$ columns if $\theta$ and $\phi$ are $i$ th associates.

4. Deleting the last row of the design, every pair of treatments $(\theta, \phi)$ occurs together in $v_{i}$ columns if $\theta$ and $\phi$ are $i$ th associates.

5. Every ordered pair of treatments $(\theta, \phi)$ occurs together in successive periods in $\lambda_{i}$ columns if $\theta$ and $\phi$ are $i$ th associates.

6. For every pair of treatments $(\theta, \phi)$ the number of columns in which $\phi$ occurs when $\theta$ is in the last row is the same as the number of columns in which $\theta$ occurs when $\phi$ is in the last row.

The parameters satisfy the following relationships:

$b=v t, \sum n_{i} \mu_{i}=t k(k-1), \sum n_{i} \lambda_{i}=t(k-1), \sum n_{i} v_{i}=t(k-1)(k-2)$

where, $n_{i}$ 's $(i=1,2, \ldots, m)$ denote the number of $i$ th associates under the given association scheme.

\section{Model}

The general model considered for the analysis of a cross-over design with $v$ treatments, $k$ periods and $b$ units is:

$y_{i j}=\mu+\alpha_{i}+\beta_{j}+\tau_{d(i, j)}+\rho_{d(i-1, j)}+e_{i j} ;(i=1,2, \ldots, k ; j=1,2, \ldots, b)$

where, $y_{i j}$ is the observed response, $\alpha_{i}$ is the effect due to $i$ th period, $\beta_{j}$ is the effect due to $j$ th unit, $\tau_{d(i, j)}$ is the direct effect of the treatment applied in the $i$ th period to the $j$ th unit, $\rho_{d(i-1, j)}$ is the residual effect of the treatment applied in the $(i-1)$ th period to the $j$ th unit, and $e_{i j}$ are random errors assumed to be normally distributed with zero mean and constant variance $\sigma^{2}$. Also, $\rho_{d(0, j)}=0$ for all $j$.

\section{Variances and Efficiencies}

Let $\mathrm{N}$ be the incidence matrix of direct effects $\times$ units, $\mathrm{N}^{*}$ be the incidence matrix of residual effects $\times$ units and $S$ be the incidence matrix of direct effects $\times$ residual 
effects. Then, as by Raghavarao and Blaisdell (1985) the average variances of the estimated direct and residual effects of a PBCOD are given respectively as

$$
V_{d}=\left\{\frac{2}{v-1}\right\}\left\{\sum_{i=1}^{v-1} \frac{k\left[k t(k-1)-\gamma_{i}\right]}{\left(k^{2} t-\rho_{i}\right)\left[k t(k-1)-\gamma_{i}\right)-\left(k \theta_{i}-x_{i}\right)^{2}}\right\} \sigma^{2}
$$

and

$$
V_{r}=\left\{\frac{2}{v-1}\right\}\left\{\sum_{i=1}^{v-1} \frac{k\left[k^{2} t-\rho_{i}\right]}{\left(k^{2}-\rho_{i}\right)\left[k t(k-1)-\gamma_{i}\right)-\left(k \theta_{i}-x_{i}\right)^{2}}\right\} \sigma^{2}
$$

Further, the efficiencies corresponding to direct and residual effects, respectively, as given by Raghavarao and Blaisdell (1985) and utilized in Aggarwal and Jha (2006) are

$$
E_{d}=\frac{v-1}{k^{2} t \sum\left\{\left(k^{2} t-\rho_{i}\right)-\left\{\frac{\left(2 k \theta_{i}-\rho_{i}-\gamma_{i}+t\right)^{2}}{4\left(k(k-1) t-\gamma_{i}\right)}\right\}\right\}^{-1}}
$$

and

$$
E_{r}=\frac{v-1}{k(k-1) t \sum\left\{\left(k(k-1) t-\gamma_{i}\right)-\left\{\frac{\left(2 k \theta_{i}-\rho_{i}-\gamma_{i}+t\right)^{2}}{4\left(k^{2} t-\rho_{i}\right)}\right\}\right\}^{-1}}
$$

Here, $\rho_{i}$ 's are eigenvalues of $\mathrm{NN}^{\prime}, \gamma_{i}$ 's are eigenvalues of $\mathrm{N}^{*} \mathrm{~N}^{* \prime}, \theta_{i}$ 's are eigenvalues of $\mathrm{S}$ and $x_{i}$ 's are eigenvalues of $\mathrm{N} \mathrm{N}^{*}$.

\section{Construction of PBCODs for consumer trials}

New classes of PBCOD(2)with a large number of units, particularly suited for use in consumer trials, are obtained here. This does away with the need of repetition of treatment sequences or relabeling of the treatments in consumer research trials, when the number of units is quite large.

\subsection{Designs based on PBIBD(2)}

Series 1: Construction of PBCOD using $\operatorname{PBIBD}(2)$ and OA is presented here.

Step 1: Consider a $\operatorname{PBIBD}(2)$ with parameters $v^{*}, b^{*}, k^{*}, r^{*}, \lambda_{1}{ }^{*}, \lambda_{2}{ }^{*}$, such that $k^{*}$ is prime or prime power, and number the treatments of each block from 1 to $k^{*}$ in order of their appearance in the block. 
Step 2: Consider an OA with $k^{*}$ symbols, obtained by superimposition of MOLS, and delete its first $k^{*}$ columns and first and last row to get a new array.

Step 3: Replace the symbols of the new array of step 2 with the corresponding treatments of the blocks to obtain the required design with parameters

$$
\begin{aligned}
& v=v^{*}, k=k^{*}-1, t=r^{*}\left(k^{*}-1\right), b=v t, \mu_{1}=\lambda_{1}{ }^{*}\left(k^{*}-1\right)\left(k^{*}-2\right), \mu_{2}=\lambda_{2}{ }^{*}\left(k^{*}-\right. \\
& 1)\left(k^{*}-2\right), v_{1}=\lambda_{1}{ }^{*}\left(k^{*}-2\right)\left(k^{*}-3\right), v_{2}=\lambda_{2}{ }^{*}\left(k^{*}-2\right)\left(k^{*}-3\right), \lambda_{1}=\lambda_{1}{ }^{*}\left(k^{*}-2\right), \\
& \lambda_{2}=\lambda_{2}{ }^{*}\left(k^{*}-2\right) .
\end{aligned}
$$

\section{Example 1:}

Consider a PBIBD(2) with parameters $v^{*}=6, b^{*}=3, k^{*}=4, r^{*}=2, \lambda_{1}{ }^{*}=2, \lambda_{2}{ }^{*}=1$ of Clatworthy (1973).

The design is

$\begin{array}{lll}1 & 2 & 3 \\ 4 & 5 & 6 \\ 2 & 3 & 1 \\ 5 & 6 & 4\end{array}$

The array obtained by deleting first four columns and first and last row of OA with four symbols, constructed by superimposition of MOLS, is

$\begin{array}{llllllllllll}0 & 1 & 2 & 3 & 0 & 1 & 2 & 3 & 0 & 1 & 2 & 3 \\ 1 & 0 & 3 & 2 & 2 & 3 & 0 & 1 & 3 & 2 & 1 & 0 \\ 2 & 3 & 0 & 1 & 3 & 2 & 1 & 0 & 1 & 0 & 3 & 2\end{array}$

Identifying the treatments of each block of $\operatorname{PBIBD}(2)$ with the symbols of $\mathrm{OA}$

$\begin{array}{lll}1 & 2 & 3 \rightarrow \mathbf{0} \\ 4 & 5 & 6 \rightarrow \mathbf{1} \\ 2 & 3 & 1 \rightarrow \mathbf{2} \\ 5 & 6 & 4 \rightarrow \mathbf{3}\end{array}$

Replacing the symbols of the array, obtained by deleting rows and columns of OA, by the corresponding treatments of the blocks, the required design is

123456231564123456231564123456231564

456123564231231564123456564231456123

231564123456564231456123456123564231 
with parameters

$$
v=6, k=3, t=6, b=36, \mu_{1}=12, \mu_{2}=6, v_{1}=4, v_{2}=2, \lambda_{1}=4, \lambda_{2}=2 .
$$

The estimated variances of the direct and residual effects are given respectively as

$$
\mathrm{V}_{\mathrm{d}}=0.1726 \quad \text { and } \quad \mathrm{V}_{\mathrm{r}}=0.2788
$$

and the efficiencies for the estimation of elementary contrasts of direct and residual effects are given, respectively, as

$$
\mathrm{E}_{\mathrm{d}}=0.6436 \text { and } \mathrm{E}_{\mathrm{r}}=0.5977 \text {. }
$$

Series 2: Construction of PBCOD using MOLS and PBIBD(2) is presented here.

Step 1: Consider a $\operatorname{PBIBD}(2)$ with parameters $v^{*}, b^{*}, k^{*}, r^{*}, \lambda_{1}{ }^{*}, \lambda_{2}{ }^{*}$, such that $k^{*}$ is prime or prime power.

Step 2: Obtain a complete set of MOLS for each block of PBIBD(2) by identifying the symbols of a Latin square of order $k^{*}$ with the treatments occurring in blocks of $\operatorname{PBIBD}(2)$ in that order.

Step 3: Place all the MOLS together to obtain the required design with parameters

$$
\begin{aligned}
& v=v^{*}, k=k^{*}, t=r^{*}\left(k^{*}-1\right), b=v t, \mu_{1}=\lambda_{1}{ }^{*} k^{*}\left(k^{*}-1\right), \mu_{2}=\lambda_{2}{ }^{*} k^{*}\left(k^{*}-1\right), v_{1}= \\
& \lambda_{1}{ }^{*}\left(k^{*}-1\right)\left(k^{*}-2\right), v_{2}=\lambda_{2}{ }^{*}\left(k^{*}-1\right)\left(k^{*}-2\right), \lambda_{1}=\lambda_{1}{ }^{*}\left(k^{*}-1\right), \lambda_{2}=\lambda_{2}{ }^{*}\left(k^{*}-1\right) .
\end{aligned}
$$

Example 2: Consider the following $\operatorname{PBIBD}(2)$ with parameters

$$
v^{*}=6, b^{*}=3, k^{*}=4, r^{*}=2, \lambda_{1}{ }^{*}=2, \lambda_{2}{ }^{*}=1
$$

of Clatworthy (1973)

$\begin{array}{lcc}\text { Block I } & \text { Block II } & \text { Block III } \\ 1 & 2 & 3 \\ 4 & 5 & 6 \\ 2 & 3 & 1 \\ 5 & 6 & 4\end{array}$

The MOLS of treatments of each block, by identifying the treatments of the blocks in order of their appearance, with the symbols of Latin square are 
MOLS of block I

\begin{tabular}{llll|lllll|lll}
1 & 4 & 2 & 5 & 1 & 4 & 2 & 5 & 1 & 4 & 2 & 5 \\
4 & 1 & 5 & 2 & 2 & 5 & 1 & 4 & 5 & 2 & 4 & 1 \\
2 & 5 & 1 & 4 & 5 & 2 & 4 & 1 & 4 & 1 & 5 & 2 \\
5 & 2 & 4 & 1 & 4 & 1 & 5 & 2 & 2 & 5 & 1 & 4
\end{tabular}

MOLS of block II

\begin{tabular}{llll|lllll|lll}
2 & 5 & 3 & 6 & 2 & 5 & 3 & 6 & 2 & 5 & 3 & 6 \\
5 & 2 & 6 & 3 & 3 & 6 & 2 & 5 & 6 & 3 & 5 & 2 \\
3 & 6 & 2 & 5 & 6 & 3 & 5 & 2 & 5 & 2 & 6 & 3 \\
6 & 3 & 5 & 2 & 5 & 2 & 6 & 3 & 3 & 6 & 2 & 5
\end{tabular}

MOLS of block III

\begin{tabular}{llll|lllll|lll}
3 & 6 & 1 & 4 & 3 & 6 & 1 & 4 & 3 & 6 & 1 & 4 \\
6 & 3 & 4 & 1 & 1 & 4 & 3 & 6 & 4 & 1 & 6 & 3 \\
1 & 4 & 3 & 6 & 4 & 1 & 6 & 3 & 6 & 3 & 4 & 1 \\
4 & 1 & 6 & 3 & 6 & 3 & 4 & 1 & 1 & 4 & 3 & 6
\end{tabular}

Placing all the MOLS together, the required design is

142514251425253625362536361436143614 415225145241526336256352634114364163

251452414152362563525263143641636341

524141522514635252633625416363411436

with parameters

$$
v=6, k=4, t=6, b=36, \mu_{1}=24, \mu_{2}=12, v_{1}=12, v_{2}=6, \lambda_{1}=6, \lambda_{2}=3 .
$$

The estimated variances of the directand residual effects are given respectively as

$$
\mathrm{V}_{\mathrm{d}}=0.1035 \quad \text { and } \quad \mathrm{V}_{\mathrm{r}}=0.1446
$$

and the efficiencies for the estimation of elementary contrasts of direct and residual effects are given, respectively, as

$$
\mathrm{E}_{\mathrm{d}}=0.8053 \quad \text { and } \quad \mathrm{E}_{\mathrm{r}}=0.7682 .
$$

Series 3: Construction of PBCOD using Williams' square and $\operatorname{PBIBD}(2)$ is presented here. 
Step 1: Take a block of PBIBD(2) with parameters $v^{*}, b^{*}, k^{*}, r^{*}, \lambda_{1}{ }^{*}, \lambda_{2}{ }^{*}$ and select $\left(k^{*}\right.$ - 1) treatments from it at a time cyclically. This gives $k^{*}$ new sequences from the block.

Step 2: Repeat step 1 for all blocks of $\operatorname{PBIBD}(2)$.

Step 3: Develop each of the $b^{*} k *$ new sequences obtained as Williams' square (one square if $p$ is even and two squares if $p$ is odd) to obtain the following two series of designs with respective parameters.

Series 3(a): $v=v^{*}, k=\left(k^{*}-1\right), t=r^{*}\left(k^{*}-1\right), b=v t, \mu_{1}=\lambda_{1}{ }^{*}\left(k^{*}-1\right)\left(k^{*}-2\right), \mu_{2}=$ $\lambda_{2}{ }^{*}\left(k^{*}-1\right)\left(k^{*}-2\right), v_{1}=\lambda_{1}{ }^{*}\left(k^{*}-2\right)^{2}, v_{2}=\lambda_{2}{ }^{*}\left(k^{*}-2\right)^{2}, \lambda_{1}=\lambda_{1}{ }^{*}\left(k^{*}-2\right), \lambda_{2}=\lambda_{2}{ }^{*}\left(k^{*}-2\right)$.

Series 3(b): $v=v^{*}, k=\left(k^{*}-1\right), t=2 r^{*}\left(k^{*}-1\right), b=v t, \mu_{1}=2 \lambda_{1}{ }^{*}\left(k^{*}-1\right)\left(k^{*}-2\right), \mu_{2}=$ $2 \lambda_{2}{ }^{*}\left(k^{*}-1\right)\left(k^{*}-2\right), v_{1}=2 \lambda_{1}{ }^{*}\left(k^{*}-2\right)^{2}, v_{2}=2 \lambda_{2}{ }^{*}\left(k^{*}-2\right)^{2}, \lambda_{1}=2 \lambda_{1}{ }^{*}\left(k^{*}-2\right), \lambda_{2}=2 \lambda_{2}{ }^{*}\left(k^{*}\right.$ $-2)$.

Example 3: Consider the following $\operatorname{PBIBD}(2)$ with parameters

$$
v^{*}=6, b^{*}=3, k^{*}=4, r^{*}=2, \lambda_{1}{ }^{*}=2, \lambda_{2}{ }^{*}=1
$$

of Clatworthy (1973)

$\begin{array}{lcc}\text { Block I } & \text { Block II } & \text { Block III } \\ 1 & 2 & 3 \\ 4 & 5 & 6 \\ 2 & 3 & 1 \\ 5 & 6 & 4\end{array}$

Let $p=3$. Thus, the new sequences obtained from the blocks of size 4 are

\begin{tabular}{llll|lllll|lll}
1 & 4 & 2 & 5 & 2 & 5 & 3 & 6 & 3 & 6 & 1 & 4 \\
4 & 2 & 5 & 1 & 5 & 3 & 6 & 2 & 6 & 1 & 4 & 3 \\
2 & 5 & 1 & 4 & 3 & 6 & 2 & 5 & 1 & 4 & 3 & 6
\end{tabular}

Developing each of these sequences as Williams square, we get the required design with parameters

$$
v=6, k=4, t=12, b=72, \mu_{1}=24, \mu_{2}=12, v_{1}=8, v_{2}=4, \lambda_{1}=4, \lambda_{2}=2 \text {. }
$$

The estimated variances of the direct and residual effects are given respectively as

$$
\mathrm{V}_{\mathrm{d}}=0.0863 \quad \text { and } \quad \mathrm{V}_{\mathrm{r}}=0.1394
$$


and the efficiencies for the estimation of elementary contrasts of direct and residual effects are given, respectively, as

$$
E_{d}=0.6436 \quad \text { and } \quad E_{r}=0.5977 \text {. }
$$

\subsection{Designs based on Triangular association scheme}

Series 4: Construction of PBCOD using MOLS and Triangular association scheme is presented here.

Step 1: Consider a triangular association scheme with $v=n(n-1) / 2$ treatments such that $2(n-1)$ is prime power, where $n$ is the order of the triangular scheme array $(n>3$ and $n \neq 6)$. Obtain sequences of treatments comprising of first associates of a treatment; different sequences for different treatments.

Step 2: Obtain complete set of MOLS of order 2(n-2) for each of the sequences by identifying the symbols of Latin squares as the treatments in the sequences.

Step 3: Place all the MOLS together to get the required design with parameters

$$
\begin{aligned}
& v=n(n-1) / 2, k=2(n-2), t=2(n-2)(2 n-5), b=v t, \mu_{1}=2(n-2)^{2}(2 n-5), \mu_{2}=8(n- \\
& \text { 2) }(2 n-5), v_{1}=2(n-2)(n-3)(2 n-5), v_{2}=8(n-3)(2 n-5), \lambda_{1}=(n-2)(2 n-5), \lambda_{2}=4(2 n- \\
& \text { 5). }
\end{aligned}
$$

Series 5: Construction of PBCOD using OA and triangular association scheme is presented here.

Step 1: Consider a triangular association scheme with $v=n(n-1) / 2$ treatmentssuch that $2(n-1)$ is prime power, where $n$ is the order of the triangular scheme array ( $n>3$ and $n \neq 6$ ). Obtain sequences of treatments comprising of first associates of a treatment; different sequences for different treatments.

Step 2: Consider an OA with 2(n-2) symbols, obtained by superimposition of MOLS, and identify the symbols with the treatments of the sequences.

Step 3: Delete the first 2(n-2) columns and first and last row of the OA to get a new array.

Step 4: Replace the symbols of the new array of step 3 with the corresponding treatments of the sequences to get the required design with parameters 


$$
\begin{aligned}
& v=n(n-1) / 2, k=(2 n-5), t=(2 n-4)(2 n-5), b=v t, \mu_{1}=(n-2)(2 n-5)(2 n-6), \\
& \mu_{2}=4(2 n-5)(2 n-6), v_{1}=(n-2)(2 n-6)(2 n-7), v_{2}=4(2 n-6)(2 n-7), \lambda_{1}=(n-2)(2 n- \\
& 6), \lambda_{2}=4(2 n-6) .
\end{aligned}
$$

Series 6: Construction of PBCOD using first associates of Triangular association scheme is presented here.

Step 1: Consider a triangular association scheme with $v=n(n-1) / 2$ treatments, where $n$ is the order of the triangular scheme array $(n>3$ and $n \neq 6)$. Obtain sequences of treatments comprising of first associates of a treatment; different sequences for different treatments.

Step 2: From a given sequence, select $\left(n_{1}-1\right)$ treatments at a time cyclically, where $n_{l}=2(n-2)$. This gives $n_{l}$ new sequences.

Step 3: Repeat step 2 for all the $v$ sequences.

Step 4: Develop each of the $v n_{l}$ new sequences obtained as Williams' square, for odd case, to obtain the required design with parameters

$v=n(n-1) / 2, k=(2 n-5), t=4(n-2)(2 n-5), b=v t, \mu_{1}=4(n-2)(n-3)(2 n-$

5), $\mu_{2}=16(n-3)(2 n-5), v_{1}=4(n-2)(n-3)(2 n-7), v_{2}=16(n-3)(2 n-7)$, $\lambda_{1}=4(n-2)(n-3), \lambda_{2}=16(n-3)$.

\subsection{Designs based on Latin square association scheme}

Series 7: Construction of PBCOD using MOLS and Latin square type association scheme is presented here.

Step 1: Consider a Latin square type association scheme with $n^{2}$ treatments such that $2(n-1)$ is prime power, where $n$ is the order of the association scheme array $(n>2$ and $n \neq 4)$. Obtain sequences of treatments comprising of first associates of a treatment; different sequences for different treatments.

Step 2: Obtain complete set of MOLS of order 2(n-1) for each sequence, by identifying the symbols of Latin squares as the treatments of the sequences.

Step 3: Place all the MOLS together to get the required design with parameters

$v=n^{2}, k=2(n-1), t=2(n-1)(2 n-3), b=v t, \mu_{1}=2(n-1)(n-2)(2 n-3), \mu_{2}=4(n-$ $1)(2 n-3), v_{1}=2(n-2)^{2}(2 n-3), v_{2}=4(n-2)(2 n-3), \lambda_{1}=(n-2)(2 n-3), \lambda_{2}=2(2 n-$ $3)$. 
Series 8: Construction of PBCOD using OA and Latin square type association scheme is presented here.

Step 1: Consider a Latin square type association scheme with $n^{2}$ treatments such that $2(n-1)$ is prime power, where $n$ is the order of the association scheme array $(n \neq 4)$. Obtain sequences of treatments comprising of first associates of a treatment; different sequences for different treatments.

Step 2: Consider an OA with 2(n-1) symbols, obtained by superimposition of MOLS, and identify the treatments of the sequences with its symbols.

Step 3: Delete first 2(n-1) columns and first and last row of this OA to get a new array.

Step 4: Replace the symbols of the new array of step 3 with the corresponding treatments of the sequences to get the required design with parameters

$v=n^{2}, k=(2 n-3), t=2(n-1)(2 n-3), b=v t, \mu_{1}=2(n-2)^{2}(2 n-3), \mu_{2}=4(n-$

2)(2n-3), $v_{l}=2(n-2)^{2}(2 n-5), v_{2}=4(n-2)(2 n-5), \lambda_{l}=2(n-2)^{2}, \lambda_{2}=4(n-2)$.

Series 9: Construction of PBCOD using first associates of Latin square type association scheme is presented here.

Step 1: Consider a Latin square association scheme with $v=n^{2}$ treatments and obtain sequences of treatments comprising of first associates of treatments; different sequences for different treatments.

Step 2: From a given sequence, select $n_{l}$ treatments at a time cyclically, where $n_{l}=$ $2(n-1)$. This gives $n_{l}$ new sequences.

Step 3: Repeat step 2 for all the $v$ sequences.

Step 4: Develop each of the $v n_{l}$ new sequences obtained as Williams' square, for odd case, to obtain the required design with parameters

$$
\begin{aligned}
& v=n^{2}, k=(2 n-3), t=4(n-1)(2 n-3), b=v t, \mu_{1}=4(n-2)^{2}(2 n-3), \mu_{2}= \\
& 8(n-2)(2 n-3), v_{1}=4(n-2)^{2}(2 n-5), v_{2}=8(n-2)(2 n-5), \lambda_{1}=4(n-2)^{2}, \lambda_{2}= \\
& 8(n-2) .
\end{aligned}
$$

Series 10: Construction of PBCOD using Latin square association scheme is presented here.

Step 1: Consider a Latin square association scheme with $v=n^{2}$ treatments and obtain sequences of treatments occurring as its rows and columns; one sequence for each row and each column. 
Step 2: Develop each of the sequences as Williams' square (one square if $n$ is even and two squares if it is odd).

Step 4: Repeat each of the squares $(n-1)^{2}$ times.

Step 5: Add a row of second associates of the treatments occurring in the last row of the squares to obtain the following two series of designs with respective parameters.

Series 10(a): $v=n^{2}, k=n+1, t=2(n-1)^{2}, b=v t, \mu_{1}=(n-1)\left(n^{2}-n+2\right), \mu_{2}=4(n-1)$, $v_{1}=n(n-1)^{2}, v_{2}=0, \lambda_{1}=(n-1)^{2}, \lambda_{2}=2$.

Series 10(b): $v=n^{2}, k=n+1, t=4(n-1)^{2}, b=v t, \mu_{1}=2(n-1)\left(n^{2}-n+2\right), \mu_{2}=8(n-1)$, $v_{1}=2 n(n-1)^{2}, v_{2}=0, \lambda_{1}=2(n-1)^{2}, \lambda_{2}=4$.

The designs for each of the series, along with their variances and efficiencies of the direct and residual effects, are presented in the following table.

Table 1: Variances and efficiencies of the estimated direct and residual effects

\begin{tabular}{|c|c|c|c|c|c|}
\hline $\begin{array}{c}\text { Series } \\
\text { No. }\end{array}$ & $\begin{array}{c}\text { Parameters } \\
\left(\boldsymbol{v}, \boldsymbol{k}, \boldsymbol{t}, \boldsymbol{b}, \boldsymbol{\mu}_{\boldsymbol{1}}, \boldsymbol{\mu}_{\boldsymbol{2}}, \boldsymbol{v}_{\boldsymbol{l}}, \boldsymbol{v}_{\mathbf{2}}, \boldsymbol{\lambda}_{\boldsymbol{l}}, \boldsymbol{\lambda}_{\mathbf{2}}\right)\end{array}$ & $\begin{array}{c}\text { Variance } \\
\text { of the } \\
\text { estimated } \\
\text { direct } \\
\text { effects }\end{array}$ & $\begin{array}{c}\text { Variance } \\
\text { of the } \\
\text { estimated } \\
\text { residual } \\
\text { effects }\end{array}$ & $\begin{array}{c}\text { Efficiency } \\
\text { of the } \\
\text { direct } \\
\text { effect } \\
\text { estimates }\end{array}$ & $\begin{array}{c}\text { Efficiency } \\
\text { of the } \\
\text { residual } \\
\text { effect } \\
\text { estimates }\end{array}$ \\
\hline 1 & $(6,3,6,36,12,6,4,2,2,4)$ & 0.1726 & 0.2788 & 0.6436 & 0.5977 \\
\hline 2 & $(6,4,6,36,24,12,12,6,6,3)$ & 0.1035 & 0.1446 & 0.8053 & 0.7682 \\
\hline 3 (a) & $(8,4,20,160,24,36,12,18,6,9)$ & 0.0320 & 0.0445 & 0.7812 & 0.7494 \\
\hline 3 (b) & $(6,3,12,72,24,12,8,4,4,2)$ & 0.0863 & 0.1394 & 0.6436 & 0.5977 \\
\hline 4 & $(6,4,12,72,24,48,12,24,4,8)$ & 0.0885 & 0.1257 & 0.4709 & 0.4418 \\
\hline 5 & $(6,3,12,72,12,24,4,8,2,4)$ & 0.0863 & 0.1394 & 0.6436 & 0.5977 \\
\hline 6 & $(6,3,24,144,24,48,8,16,4,8)$ & 0.0863 & 0.1394 & 0.6436 & 0.5977 \\
\hline 7 & $(9,4,12,108,12,24,6,12,2,4)$ & 0.0547 & 0.0753 & 0.7617 & 0.7382 \\
\hline 8 & $(9,3,12,108,6,12,2,4,2,4)$ & 0.0909 & 0.1432 & 0.6111 & 0.5820 \\
\hline 9 & $(9,3,24,216,12,24,4,8,4,8)$ & 0.0455 & 0.0716 & 0.6111 & 0.5820 \\
\hline 10 (a) & $(16,5,18,288,42,12,36,0,9,2)$ & 0.0284 & 0.0374 & 0.7832 & 0.7419 \\
\hline 10 (b) & $(9,4,4,36,6,2,2,0,2,1)$ & 0.1736 & 0.2496 & 0.7202 & 0.6677 \\
\hline
\end{tabular}

\section{Conclusions}

Some new classes of PBCOD (2) are constructed using OA, PBIBD(2), MOLS and two associate class association schemes. The constructed designs are particularly useful for consumer research trials as they expanse over a large number of units and 
consumer research trials are generally conducted over a large population. The computed variances and efficiencies show that the designs are efficient for practical purposes. Also, for continuous responses, the data obtained from the designs can be suitably analysed using the method outlined in Jones and Kenward (2003, pp: 212) and in the example of analysis of cross-over designs given at Design resources Server at http://www.iasri.res.in/design/Analysis\%20of\%20data/change-overdesigns.html (accessed lastly on March 28, 2015).

\section{References:}

1. Aggarwal, M. L., and Jha, M. K. (2006). Some new series of partially balanced residual treatment effects designs. Communications in StatisticsTheory and Methods, 35:1477-1482. DOI: $10.1080 / 03610920600695824$

2. Ball, R. D. (1997). Incomplete block designs for the minimisation of order and carry-over effects in sensory analysis. Food and Quality Preference, 8:111-118.

DOI: $10.1016 / \mathrm{S} 0950-3293(96) 00026-2$

3. Blaisdell, E. A., and Raghavarao, D. (1980). Partially balanced changeover designs based on m-associate class PBIB designs. Journal of Royal Statistical Society, B42:334-338.

4. Clatworthy, W. H. (1973). Tables of two-associate-class partially balanced designs. National Bureau of Standards, Washington D. C.

5. Kunert, J., and Sailor, O. (2006). On nearly balanced designs for sensory trials. Food and Quality Preference, 17: 219-227.

DOI: 10.1016/j.foodqual.2005.05.011

6. MacFie, H. J. H., Bratchell, N., Greenhoff, K., and Vallis, L.V. (1989). Designs to balance the effect of order of presentation and first-order carryover effects in hall tests. Journal of Sensory Studies, 4:129-148. DOI: $10.1111 / j .1745-459 X .1989 . t b 00463 . x$

7. Patterson, H. D. (1951). Change-over trials. Journal of Royal Statistical Society, B13:256-271.

8. Patterson, H. D. (1952). The construction of balanced designs for experiments involving sequences of treatments. Biometrika, 39:32-48. DOI: $10.1093 /$ biomet/39.1-2.32

9. Périnel, E., and Pagés, J. (2004). Optimal nested cross-over designs in 
sensory analysis. Food and Quality Preference, 15:439-446.

DOI: 10.1016/j.foodqual.2003.07.004

10. Raghavarao, D., and Blaisdell, E. A. (1985). Efficiency bounds for partially balanced change-over designs based on m-associate class PBIB designs. Journal of Royal Statistical Society, B47:132-135.

11. Schlich, P. (1993). Uses of change-over designs and repeated measurements in sensory and consumer studies. Food Quality and Preference, 4:223-235. DOI: 10.1016/0950-3293(93)90166-4

12. Wakeling, I. N., Hasted, A., and Buck, D. (2001). Cyclic presentation order designs for consumer research. Food Quality and Preference, 12:39-46. DOI: $10.1016 / \mathrm{S} 0950-3293(00) 00027-6$

13. Wakeling, I. N., and MacFie, H. J. H. (1995). Designing consumer trials balanced for first and higher orders of carry-over effect when only a subset of k sample from t may be tested. Food and Quality Preference, 6:299-308. DOI: 10.1016/0950-3293(95)00032-1

14. Williams, E. J. (1949). Experimental designs balanced for the estimation of residual effects of treatments. Australian Journal of Science and Research, A2:149-168.

DOI: $10.1071 / \operatorname{ch} 9490149$ 